\title{
A new heating/quenching stage for melt inclusion homogenisation: hundreds of olivine grains in one experiment
}

\author{
IGOR K. NIKOGOSIAN ${ }^{1}$, JANNE M. KOORNNEEF ${ }^{1}$, \\ ONNO POSTMA $^{2}$, JACOB ARENDS ${ }^{2}$, WIM VAN DER \\ PLAS $^{2}$ AND JOOST ROSIER ${ }^{2}$
}

${ }^{1}$ Vrije Universiteit

${ }^{2}$ VU Amsterdam

Presenting Author: i.nikogosian@vu.nl

With the development of new analytical techniques, the study of olivine-hosted melt inclusions (MI) becomes a more valuable and demanded method to collect geochemical information of primary mantle-derived melts. While a decade ago analyses included major, trace and volatile elements, recently it became possible to measure radiogenic isotopes in primitive MI advancing our understanding of the mosaics of mantle source heterogeneity and its link to geodynamics [1].

Melt inclusion petrography can vary from glassy to fully crystallised. Glassy inclusions can directly be used for geochemical analysis, but the latter require heating/quenching experiments, to melt the daughter phases and homogenise the crystallised MI to a glass.

The optimal technique to homogenise $\mathrm{MI}$ is a heating/quenching stage with visual control [2]. Such a heating stage typically homogenises MIs in a single double-polished crystal, however this is a time-consuming effort if large amounts of olivine with MIs are to be studied.

Here, we present a new design heating stage, built in-house, that allows fast and easy heating/quenching of up to 100 olivine grains simultaneously at a size of 500-1000 microns. The heating stage consists of a water-cooled metallic body, an Macor housing, a Pt alloy heating element, and a Pt sample holder bucket $(1 \times 1 \mathrm{~cm})$ installed on a carrier to be easily moved in and out of the heating element enabling fast cooling rates for $\mathrm{MI}$ quenching $\left(2-3 \mathrm{sec}\right.$ down to $\left.800^{\circ} \mathrm{C}\right)$. To avoid oxidation of the host minerals during the experiment, oxygen fugacity is controlled under $\mathrm{He}$ atmosphere purified with a Ti filter at $700^{\circ} \mathrm{C}$ ( IW buffer).

The maximum temperature within the bucket monitored by a $\mathrm{Pt}_{90} \mathrm{Rd}_{10}$ thermocouple is $1450^{\circ} \mathrm{C}$. The accuracy of the temperature calibrated by various standards is $5^{\circ} \mathrm{C}$, with a temperature gradient of $15^{\circ} \mathrm{C}$ along the bucket floor. The new stage allows controlled homogenisation of hundreds of olivinehosted MI per day.

[1] Koornneef et al., 2019, Nature Communications. 10, 3237

[2] Sobolev et al., 1980, Proc. Lunar Planet. Sci. Conf. 11th, pp. $105-116$ 